\title{
New components of the mercury's perihelion precession
}

\author{
Joseph J. Smulsky
}

Institute of Earth's Cryosphere of Siberian Branch of Russian Academy of Sciences, Tyumen, Russia; Jsmulsky@mail.ru

Received 28 January 2011; revised 27 February 2011; accepted 27 March 2011.

\begin{abstract}
The velocity of perihelion rotation of Mercury's orbit relatively motionless space is computed. It is prove that it coincides with that calculated by the Newtonian interaction of the planets and of the compound model of the Sun's rotation.
\end{abstract}

Keywords: Mercury; Perihelion; Observation; Calculation; Sun's Compound Model

\section{INTRODUCTION}

There are many lasting puzzles in the Solar system the understanding of which is crucial, and that for decades and some for centuries have not received a final explanation. One such phenomenon is the precession of the perihelion of Mercury's orbit. The encyclopedia Wikipedia [1] posted an article "Tests of general relativity" of evidence confirming the General Relativity (GR) by the observations. In particular, Table 1 gives the confirmation of General Relativity in the perihelion of Mercury. The data in Table 1 are well known in theoretical physics [2-5]. However, due to lack of awareness of theoretical physicists, several lines of this table are incorrect. Therefore, we consider the rotation of the perihelion of Mercury in detail.

\section{OBSERVED MOTION OF THE VERNAL EQUINOX}

The rotation of the Mercury perihelion can be determined as a result of the analysis of changes of several parameters of planets orbits. For this purpose we shall consider, what changes of the planets orbits occur and from which points the readout of angles is carried out. On Figure 1 in heliocentric equatorial system of coordinates $x y z$ the orbit plane of planet (Mercury) draws an arc of circle $D A B$ on celestial sphere, and the projection of the orbit's perihelion is marked by point $B$. The motionless planes of equator $A_{0} A_{0}^{\prime}$ and ecliptics $E_{0} E_{0}^{\prime}$ are fixed on the certain epoch $T_{S}$, for example, 1950.0 or 2000.0. Other planes of equator $A A^{\prime}$, of ecliptics $E E^{\prime}$ and of Mercury $D A B$ in epoch $T$ are moving in space.

The angles between planes are submitted on Figure 2, there corresponds Figure 2(a) of our work [6] in which the results are given for Mars, but they are fair for any planet, including Mercury. As the planes of Earth equator $A A^{\prime}$ and of Earth orbit $E E^{\prime}$ on Figure 2 move in space, therefore the vernal equinox point $\gamma$ retreats on an arc $\gamma_{2} \gamma$ from motionless equator plane $A_{0} A_{0}^{\prime}$ with velocity

$$
p_{c}=5025^{\prime \prime} 641+2 " 223 \cdot T_{t},
$$

where $p_{c}$ - velocity in arcsec/century, $T_{t}$ - time in tropical centuries from epoch of 1900.0. It needs to note, that in geocentric system the Sun passes point $\gamma$ at spring, and in heliocentric system the Earth passes point $\gamma$ at autumn.

The Eq.1 is derived by S. Newcomb [7] as a result of approximation of observation data on an interval several hundreds years. It gives velocity of retreating of point $\gamma$ from a motionless equator plane $A_{0} A_{0}^{\prime}$, which is equal to $-5026.75 \mathrm{arcsec} / \mathrm{century}$ for 1950.0 and -5027.86 arcsec/century for 2000.0 . As the point $\gamma$ moves clockwise therefore the velocity is written down with this sign

Table 1. Sources of the precession of perihelion for Mercury according to the encyclopedia Wikipedia [1].

\begin{tabular}{cc}
\hline $\begin{array}{c}\text { Amount } \\
\text { (arcsec/Julian century) }\end{array}$ & Cause \\
$5028.83 \pm 0.04[2]$ & $\begin{array}{c}\text { Coordinate (due to the precession of } \\
\text { the equinoxes) } \\
530[3]\end{array}$ \\
0.0254 & $\begin{array}{c}\text { Gravitational tugs of the other planets } \\
\text { Oblateness of the Sun } \\
\text { (quadrupole moment) }\end{array}$ \\
$42.98 \pm 0.04[4]$ & General relativity \\
5603.24 & Total \\
$5599.7[5]$ & Observe \\
$-3.54(-0.0632 \%)$ & Discrepancy \\
\hline
\end{tabular}




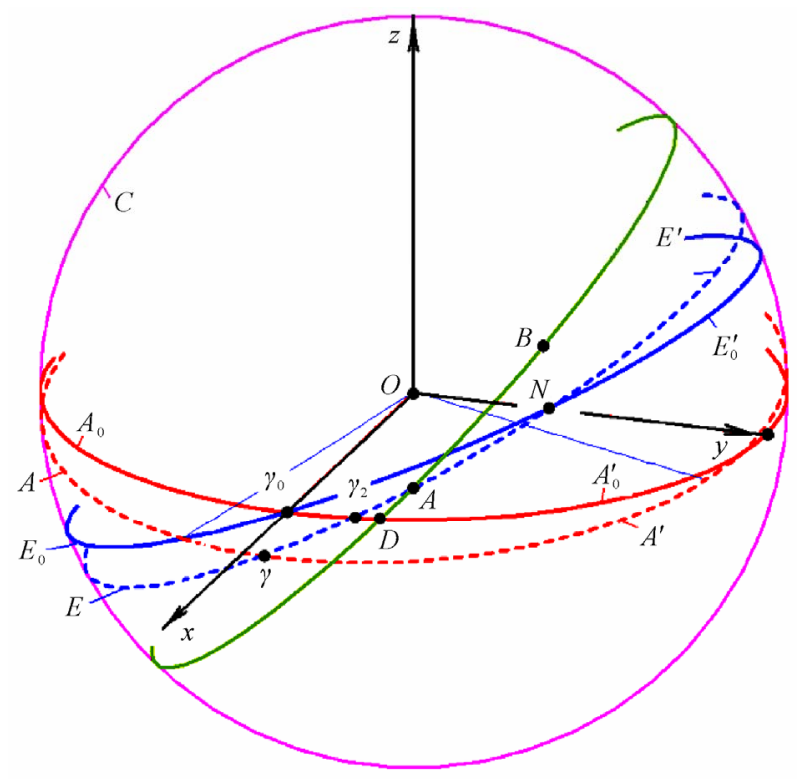

Figure 1. The basic planes on celestial sphere $C: A_{0} A_{0}^{\prime}$ - a motionless plane of the Earth's equator for epoch $T_{S} ; E_{0} E_{0}^{\prime}$ - a motionless plane of the Earth's orbit for epoch $T_{S}$ (a plane of motionless ecliptic); $A A^{\prime}$ - a mobile plane of the Earth's equator in epoch $T ; E E^{\prime}$ - a mobile plane of the Earth's orbit in epoch $T$ (the inclination for presentation is increased); $\gamma_{0}$ - vernal equinox point of epoch $T_{S} ; \gamma$ - a point on a line of crossing of mobile equator in epoch $T$ with a mobile ecliptic (vernal equinox point in epoch $T$ ); $D A B$ - a plane of the Mercury's orbit in epoch $T$.

“-". Note that in modern treatment of observation data by J. L. Simon et al. [8], the velocity of removal of the point $\gamma$ is -5028.82 arcsec per century.

Thus, the number 5028.83 arcsec per century in the first row in the Table 1 represents the motion of the vernal equinox $\gamma$ relative to the motionless space.

\section{THE RELATIVE VELOCITY OF THE PERIHELION ON THE OBSERVATIONS}

In Astronomy the moton of perihelion point $B$ is defined by a longitude $\pi_{a}$, which as result of approximation of observation data S. Newcomb [7] represents as a polynomial of the third power of time:

$$
\begin{aligned}
\pi_{a}= & 334^{\circ} 13^{\prime} 05^{\prime \prime} 53+6626^{\prime \prime} 73 \cdot T_{j}, \\
& +0^{\prime \prime} 4675 \cdot T_{j}^{2}-0^{\prime \prime} 0043 \cdot T_{j}^{3},
\end{aligned}
$$

where $T_{j}$ - time counted in Julian centuries for 36525 days from fundamental epoch 1900.0.

Value $\pi_{\mathrm{a}}$ represents the sum of two different arcs (see Figure 2)

$$
\pi_{a}=\gamma A+A B
$$

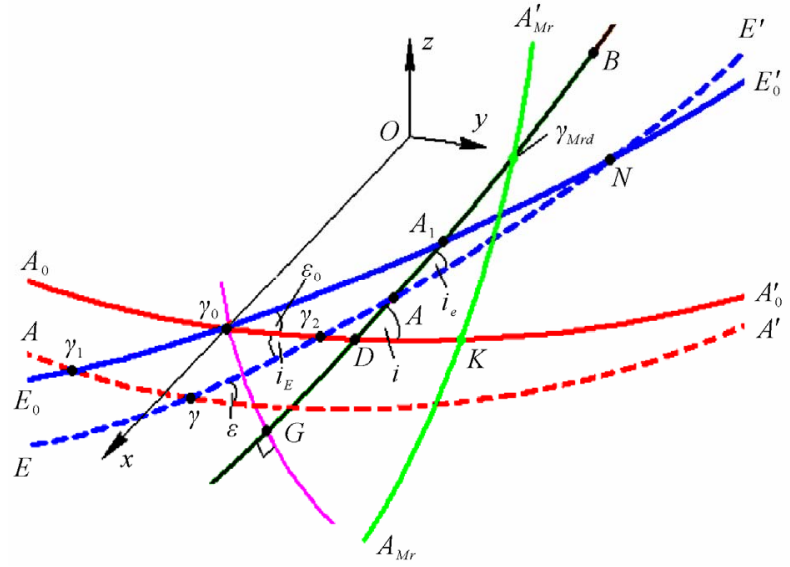

Figure 2. The parts of the Mercury's perihelion rotation on the celestial sphere. The designations of planes it is given on Figure 1; $\gamma_{0} G$ - an arc of the big circle, which is perpendicular to plane of the Mercury's orbit $G D A B ; B$ - the heliocentric projection of the Mercury's perihelion to celestial sphere; $A$ - ascending node of the Mercury's orbit on mobile ecliptic; $D$ ascending node of the Mercury's orbit on motionless equator of epoch $T_{S}$; the parameters of the Mercury's orbit in inertial equatorial reference frame: $\varphi_{\Omega}=\gamma_{0} D ; \varphi_{p}=D B ; i=\angle \gamma_{0} D G ; i_{E}$ inclination of the Earth mobile orbit (mobile ecliptic); and in mobile ecliptic system $\Omega_{a}=\gamma A ; \omega_{a}=A B ; \pi_{a}=\gamma A+A B=\Omega_{a}+$ $\omega_{a} ; i_{e a}=i_{e}=\angle \gamma A G$; index " $e$ " - the angles relatively to mobile ecliptic; index " $a$ " - by results of approximation of observation data.

where arc $\gamma A=\Omega_{a}$ refers as longitude of ascending node of Mercury's orbit.

From the Eq.2 the velocity of perihelion rotation on a way of arcs $\gamma A+A B$ is equal 5602.9 arcsec/century for 1950.0 and 5601.9 arcsec/century for 2000.0. For elements of J. L. Simon et al. [8] it is equal to 5603.0 arcsec par century to 2000.0. From Figure 2 it is seen, that at definition of perihelion point $B$ by value $\pi_{a}$, in velocity of perihelion will enter: 1) velocity of movement of point $\gamma$ on mobile ecliptic $\left.E E^{\prime} ; 2\right)$ velocity of displacement of point $A$ of mobile ecliptic $E E^{\prime}$ due to its rotation around point $N$ and 3) velocity of displacement of ascending node $A$ of Mercury orbit $G D A B$ on mobile ecliptic $E E^{\prime}$, caused by rotation of plane $G D A B$.

Thus, the value 5599.7 arcsec per century in the 6-th row of Table 1 gives the velocity of the perihelion rotation from the moving point $\gamma$ of the vernal equinox with the inclusion of velocities of change of the ecliptic and of the Mercury orbit. It is slightly differs from the values $5602 \div 5603$ arcsec per century determined by the elements of S. Newcomb [7] and J. L. Simon et al. [8]. This velocity is not absolute but relative. In addition, as shown above, it includes the rates of change of the ecliptic and the orbit of Mercury. 


\section{ABSOLUTE VELOCITY OF THE PERIHELION ON OBSERVATIONS}

To these velocities did not affect the velocity of perihelion movement, it should be counted from a motionless point. As such point we have taken the point $G$, which is on crossing of the circle $G D A B$ with perpendicular circle to it $\gamma_{0} G$. In work [6] we have derived the formula (29) for arc $G B$ which depends on parameters of mobile orbit planes of the Earth $\left(E E^{\prime}\right)$ and of the Mercury $(G D A B)$ and have the following form:

$$
\begin{aligned}
\varphi_{p 0}^{a}=G B & =\pi_{a}-\Omega_{a} \\
& +\arcsin \left[\sin i_{E a} \sin \left(\Omega_{a}-\gamma \gamma_{2}\right) / \sin i^{a}\right] \\
& +\arccos \left[\cos \varphi_{\Omega}^{a} /\left(1-\left(\sin _{\Omega}^{a} \sin i^{a}\right)^{2}\right)^{0.5}\right]
\end{aligned}
$$

The designations are given in the caption signature to Figure 2. For angles $\varphi_{0}^{a}$ and $i_{E a}$ in work [6] are also given formulas, which depend on the ecliptic angles of orbits: $\Omega_{a}, i_{e a}$ etc. As a result of approximation of observation data S. Newcomb [7] has presented ecliptic angles as polynomials of the third degree on time which example is the Eq.2. J. L. Simon et al. [8] have modified Newcomb's results for epoch of 2000.0 and have given as polynomials of 6-th degree.

The Eq.4 gives velocity of perihelion rotation of Mercury orbit relatively motionless space which equals $582.05 \mathrm{arcsec} /$ century for 1950.0 and 583.15 for 2000.0 $[9,10]$. It is velocity of perihelion rotation according to observation. Due to orbits elements by J. L. Simon et al. [8] it equals 582.53 arcsec/century for 2000.0. It is absolute velocity of the perihelion on observations data.

Thus, in the Table 1 the velocity of the perihelion rota- tion on observation data does not been determined. It is equal to $582-583$ arcsec per century relatively to the motionless space.

\section{THE ROTATION OF PERIHELION FOR MERCURY UNDER ACTION OF THE PLANETS AND THE SUN}

\subsection{Gravitational Tugs of the Other Planets}

The interaction of the Solar system bodies under the Newton law of gravitation results in change of their orbits (see Figure 3), including the rotation of perihelion. We have developed a refined approach for integrating the equations of celestial and space dynamics, on which is created the program Galactica. In many of our works, for example $[6,11]$, the periods and the amplitudes of the changes of the planets orbits elements are received at dif- ferent time spans, including up to 100 million years. In these calculations the bodies are considered as mate- rial points, which interact under Newton's law of gravity. On Figure 3 in span of 7 thousand years the points 1 show the evolution of the elements of the Mercury orbit, ob- tained with the Galactica. For comparison, the approxima- tions of observation data, made in 1895 by S. Newcomb [7] (line 2) and a hundred years later by J. L. Simon et al. [8] (line 3) are given. The evolution of the Mercury perihelion angle relative to the motionless point $G$ in Figure 2 is seen on points 1 on Figure 3 for element $\varphi_{p 0}$. Its velocity is 529.86 arcsec per century. This differs from the observation data at 53 arcsec per century, but not at 43 arcsec per century, as previously thought.

For finding out of the reason of difference of calculated on Newton interaction and according to observation of the velocity of Mercury perihelion rotation we have carried out various researches. First, we have established, that such difference of velocity of perihelion rotation is presented only for the Mercury, which is the closest planet to the Sun. Second, the calculated on Newton interaction other parameters of Mercury orbit and velocity of their change practically coincided with the data of observation $[9,10]$.

\subsection{Influence of Finite Propagation Speed of Gravity}

We investigated influence of gravity propagation velocity on results of interaction of two bodies. The General Theory of Relativity was created to take into account final velocity of gravity. A. Einstein has based it on the equations and results received by Paul Gerber. Paul Gerber has thought up such mechanism of gravity propagation with velocity of light that it is explained rotation of perihelion in 43 arcsec/century [12]. However, as we have shown in paper [10], this mechanism is proved by nothing and is erroneous. Besides as it is above shown, the difference of calculations on Newton interaction and observation is equal to not 43 arcsec /century but 53 arcsec/century.

In nature only one mechanism of interaction propagation with speed of light is known: it is mechanism of propagation of electromagnetic interaction. From experimental laws of electromagnetism we have derived equation [13-15] for force of interaction of two particles with charges $q_{1}$ and $q_{2}$ :

$$
\boldsymbol{F}(\boldsymbol{r}, \boldsymbol{v})=\frac{k\left(1-\beta^{2}\right) \boldsymbol{r}}{\left\{r^{2}-[\boldsymbol{\beta} \times \boldsymbol{r}]^{2}\right\}^{3 / 2}},
$$

where $k=k_{e}=q_{1} q_{2} / \varepsilon, \quad \boldsymbol{\beta}=\boldsymbol{v} / c_{1}, \boldsymbol{r}$ and $\boldsymbol{v}$ - distance and velocity of one particle relatively another; $\varepsilon$ - dielectric permeability of media between particles, and $c_{1}$ - speed of light in media.

Apparently from (5), at not instant interaction the force 


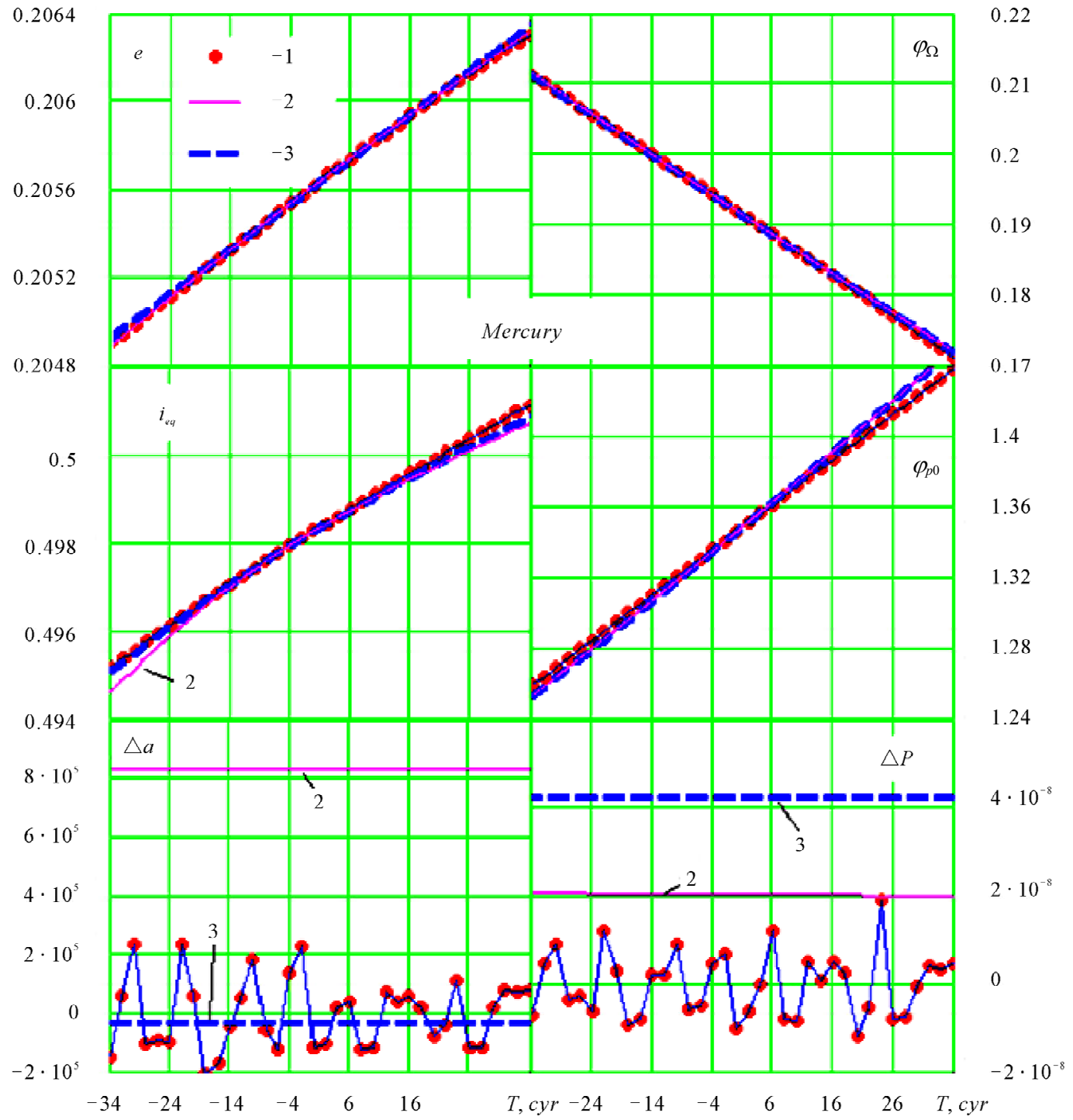

Figure 3. The secular changes in orbital elements of Mercury in the interval 7 thousand years and oscillations of semi-major axis $(a)$ and orbital period $(P): \Delta a$ and $\Delta P$ are deviations from the means for 7 thousand years; 1 is result of the numerical solution of the program Galactica; 2 is secular variations on Newcomb S., 3 is secular variations on Simon J.L. et al.; $e$ is eccentricity; $i$ is the angle of inclination of the orbital plane to the plane of the motionless Earth's equator at the epoch $2000.0, \varphi_{\Omega}$ is the angular position of the ascending node of the orbit from the motionless equatorial plane; $\varphi_{p 0}=G B$ (see Figure 2). The angles are in radians, the time $T$ in centuries from 1949 December 30.0, $\Delta a$ in meters and $\triangle P$ in years.

depends not only on distance $\boldsymbol{r}$ between particles, but also from their relative velocity $\boldsymbol{v}$. If for gravity to accept the same mechanism of interaction propagation the formula (5) will define the gravity force at $k=k_{G}=$ $-G \cdot m_{1} \cdot m_{2}$, where $m_{1}$ and $m_{2}$ - masses of interacting bodies, and $G$ - gravitational constant. With force $\boldsymbol{F}(\boldsymbol{r}, \boldsymbol{v})$ we have calculated a trajectory of movement of one body relatively another at all possible changes of an eccentricity and velocity of body in a perihelion [14-18]. In case of an elliptic orbit the perihelion rotates and the more strongly, than there is the more velocity of body in a perihelion. In such orbit the length of the semi-major axis and the period change in comparison with the orbit received at interaction of two bodies under the Newton law of gravity. The changes of the semi-major axis and the period have the same order as the change of the perihelion angle.

The calculation of rotation of Mercury perihelion at force $\boldsymbol{F}(\boldsymbol{r}, \boldsymbol{v})$ has given velocity 0.23 arcsec/century, i.e. almost in 200 times smaller than velocity 43 
arcsec/century explained by Paul Gerber [12] and accepted in GR. The conclusion from here follows that surplus of the perihelion rotation in $52 \div 53$ arcsec/century may not be explained by the mechanism of gravity interaction propagation with speed of light.

\subsection{The Precession of the Perihelion under Action of the Sun}

The explanation of surplus of perihelion rotation of the Sun oblateness now is complicated with complexities of model of interaction and absence of knowledge of distribution of the Sun density on radius and along an axis of the Sun. Therefore the executed calculations of influence of the Sun oblateness, most likely, are doubtful.

If inside the Mercury orbit there was a planet of the certain mass, it might make necessary rotation of the Mercury perihelion and at the same time not to render appreciable influence on other planets. Such planet is not present. But the Sun rotates about its axis, and the moving masses of its substance may influence Mercury the same as the planet offered above. During two centuries these ideas were put forward in various forms. However, the satisfactory solutions have not been received. In 2007 , the problem of axisymmetric gravitational n-body interaction has been solved exactly (see $[14,15,19])$. Before this solution, there was only one exact solution of the problem of bodies interaction, namely for two bodies, it was received by I. Newton 300 years ago. In works [15] and [19] the exact solution for a symmetrically located on a plane bodies has obtained for all possible cases. The bodies, as in the case of the two bodies, can move in a circle, ellipse, parabola, hyperbola and straight lines. This solution is allowed to create a compound model of the Earth's rotation (see [11,20]), in which a part of the Earth's mass is distributed between the bodies symmetrically located in the equator plane of Earth. The orbital plane of one of these bodies simulates the evolution of the Earth's equatorial plane under the action of other Solar system bodies.

With assistance of the compound model of the Sun's rotation (see $[9,10,21]$ ) it is considered the inverse problem: to what changes in the planets motion would such model of the Sun's rotation? The papers $[9,10]$ considered various variants for the action of the compound of the Sun's rotation with jointly action of other Solar system bodies. Appearently, that at the certain mass of periph- eral bodies of model the same velocity of rotation of perihelion may be received as observed one, i.e. 583 arcsec/century. In this case, the velocity of change of other parameters of the Mercury's orbit essentially does not change. The velocity of the Venus perihelion does not change essentially, and parameters of planets are more distant from the Sun and also change still to a lesser degree. Let's note that the compound model of the Sun rotation takes into account of the Sun oblateness and rotation of its masses.

To make sure that the additional rotation of the perihelion is due only to the compound model, it was studied the action only one compound model of the Sun's rotation (without planets) on Mercury [21]. The differential equations of motion of all bodies have been integrated numerically and have been studied the evolution of Mercury for three thousand years. In this case the rotation of its orbit is received of 53 arcsec per century, i.e. precisely the surplus, which is available at the joint influence of compound model of the Sun at the planets.

As a result of the carried out researches we shall write down the basic characteristics of rotation of Mercury perihelion in such form (see Table 2) that they could be compared to the data on Wiki site. Apparently from

Table 2. Velocity of rotation of the Mercury perihelion on observation for 2000.0 on orbit elements of S. Newcomb [7] (Ncb) and J. L. Simon et al. [8] (Sim) and on Newton interaction. To compare in round brackets - accordingly Wikipedia [1].

\begin{tabular}{|c|c|}
\hline $\begin{array}{c}\text { Amount } \\
\text { (arcsec/century) }\end{array}$ & Explanation \\
\hline \multicolumn{2}{|r|}{ On observation data } \\
\hline $\begin{array}{l}-5028.82-\mathrm{Sim} \\
(5028.83-\mathrm{Wiki})\end{array}$ & \multirow[b]{2}{*}{$\begin{array}{l}\text { Velocity of perihelion rotation relatively the mobile vernal equinox point } \gamma \text { with including velocities changes of ecliptic } \\
\text { and of Mercury orbit (according form. (2)). }\end{array}$} \\
\hline $\begin{array}{c}5601.9-\mathrm{Ncb} \\
5603.0-\mathrm{Sim} \\
(5599.7-\mathrm{Wiki})\end{array}$ & \\
\hline $\begin{array}{l}583.15-\mathrm{Ncb} \\
582.53-\mathrm{Sim}\end{array}$ & Velocity of perihelion rotation relatively motionless space (according form. (4)). \\
\hline \multicolumn{2}{|r|}{ By results of interaction under the Newton law of gravity. Velocity of rotation of a perihelion relatively motionless space. } \\
\hline $530(530-$ Wiki $)$ & Planets and the Sun interact as material points. \\
\hline
\end{tabular}


above-stated, the problem of perihelion rotation is defined by many circumstances. Here we have not mentioned a problem of reliability of observation data approximation. We have tried to state other problems clearly and with necessary explanatory that everyone might pass on this way and be convinced of our conclusions.

We have briefly outlined a number of stages of the research phenomenon of rotation of the Mercury perihelion, which were performed by computer algorithms. We used the numerical integration of the differential equations systems, a variety of calculations with geometric transformations, mathematical treatment of time series and other computer calculations. Due to them, it was found that the components of the perihelion rotation of the Mercury's orbit can be explained by the correct account of Newton's gravitational force in the interaction of the celestial bodies.

\section{CONCLUSIONS}

1) The velocity of perihelion rotation relatively motionless space accordingly observation data is equal to 583 arcsec per century.

2) The velocity of perihelion rotation relatively motionless space as a result of interaction of the planets under the Newton law of gravity is 530 arcsec per century.

3) The Newtonian interaction of planets and of the compound model of the Sun's rotation gives the observed Mercury's perihelion precession.

\section{ACKNOWLEDGEMENTS}

I am grateful to David Weber for being interested in the problem and his work to notify society of our received results. Many calculations in the above-mentioned studies were performed on supercomputers of Siberian Supercomputer Center of Russian Academy of Science.

\section{REFERENCES}

[1] Wikipedia. 2011.http://en.wikipedia.org/wiki/Tests_of_g eneral_relativity

[2] NASA Jet Propulsion Laboratory (2010) Astrodynamic Constants retrieved. http://ssd.jpl.nasa.gov/?constants

[3] Matzner, R.A. (2001) Dictionary of geophysics, astrophysics, and astronomy. CRC Press, Florida. http://books.google.com/?id=eez38xjCYGkC

[4] Iorio, L. (2004) On the possibility of measuring the solar oblateness and some relativistic effects from planetary ranging. http://arxiv.org/abs/gr-qc/0406041v4

[5] Chow, T.L. (2008) Gravity, black holes, and the very early universe: An introduction to general relativity and cosmology. Springer, Berlin. $\mathrm{http} / / /$ books.google.com/?id=fp9wrkMYHvMC

[6] Grebenikov, E.A. and Smulsky, J.J. (2007) Evolution of the mars orbit on time span in hundred millions years/ reports on applied mathematics. A. A. Dorodnicyn Computing Center, Russian Academy of Sciences, Moscow. (In Russian)

http://www.ikz.ru/ smulski/Papers/EvMa100m4t2.pdf

[7] Newcomb, S. (1895) The elements of the fourth inner planets and the fundamental constants of astronomy. Government Printing Office, Washington.

[8] Simon, J.L., Bretagnon, P., Chapront, J. et al. (1994) Numerical expression for precession formulae and mean elements for the moon and the planets. Astronomy \& Astrophysics, 282, 663-683.

[9] Smulsky, J.J. (2008) Compound model of rotation of the Sun and displacement of Mercury perihelion/The fundamental and applied problems of the mechanics. Proceeding of the VI All-Russian Scientific Conference, devoted 130-th anniversary of Tomsk State University and 40-th anniversary NII of Applied Mathematics and the Mechanics of Tomsk State University, University Publishing House, Tomsk. (In Russian)

http://www.ikz.ru/ smulski/Papers/ModSun51c.pdf

[10] Smulsky, J.J. (2009) Gravitation, field and rotation of mercury perihelion. Proceedings of the Natural Philosophy Alliance, 15th Annual Conference 7-11 April 2008 at the University of New Mexiko, Albuquuerque, 5, 254-260. http://www.ikz.ru/ smulski/Papers/08Smulsky2c.pdf

[11] Melnikov, V.P. and Smulsky, J.J. (2009) Astronomical theory of ice ages: New approximations. Solutions and Challenges. Academic Publishing House "GEO", Novosibirsk.

http://www.ikz.ru/ smulski/Papers/AsThAnE.pdf

[12] Gerber, P. (1898) Die raumliche und zeitliche Aubreitung der Gravitation. Zeitschrift f. Mathematik und Physik, 43, 93-104. http://bourabai.narod.ru/articles/gerber/gerber.ht m. In English: http://bourabai.kz/articles/gerber/gerber-1902.pdf.

[13] Smulsky, J.J. (1994) The new approach and superluminal particle production. Physics Essays, 7, 153-166. http://www.smul1.newmail.ru/English1/FounPhisics/NA pSup.pdf

[14] Smulsky, J.J. (1999) The theory of interaction. Publishing house of Novosibirsk University, Scientific Publishing Center of United Institute of Geology and Geophysics Siberian Branch of Russian Academy of Sciences, Novosibirsk. (In Russian).

[15] Smulsky, J.J. (2004) The theory of interaction. Publishing House "Cultural Information Bank", Ekaterinburg. (In English) http://www.smul1.newmail.ru/English1/Fou nPhisics/TVANOT1.doc

[16] Smulsky, J.J. (1995) The trajectories at interaction of two bodies, which depend on relative distance and velocity. Mathematical Modeling, 7, 111-126. (In Russian). http://www.smul1.newmail.ru/Russian1/FounPhisics/TrV 2tl.pdf

[17] Smulsky, J.J. (2002) The new fundamental trajectories: Part 1-hyperbolic/elliptic trajectories. Galilcan Electrodynamics, 13, 23-28.

[18] Smulsky, J.J. (2002) The new fundamental trajectories: Part 2-parabolic/elliptic trajectories. Galilcan Electrodynamics, 13, 47-51. http://www.smull.newmail.ru/English1/FounPhisics/NFT. pdf 
[19] Smulsky, J.J. (2003) Axisymmetrical problem of gravitational interaction of N-bodies. Mathematical Modelling, 15. 27-36. (In Russian).

http://www.smul1.newmail.ru/Russian1/IntSunSyst/Osvn b4.doc

[20] Mel'nikov, V.P., Smul'skii I.I. and Smul'skii Ya.I. (2008) Compound modeling of Earth rotation and possible implications for interaction of continents. Russian Geology and Geophysics, 49, 851-858. http://www.ikz.ru/ smulski/Papers/RGG190.pdf doi:10.1016/j.rgg.2008.04.003

[21] Smulsky, J.J. (2008) Numerical modeling of evolution of the satellite of a rotating body. In Dorodnicyn A.A., Ed., The Theoretical and Applied tasks of the Nonlinear Analysis, Russian Academy of Sciences, Moscow. (In Russian).

http://www.ikz.ru/ smulski/Papers/ModSun07c.pdf 